$\underline{\xi}=-1$

\title{
Twitter Sentiment Analysis and Visualization Using Apache Spark and Elasticsearch
}

\author{
Maragatham $\mathbf{G}^{1}$, Shobana Devi $\mathrm{A}^{2}$ \\ ${ }^{I}$ Research Supervisor, Department of Information \& Technology, SRM University, Chennai, India. \\ ${ }^{2}$ Research Scholar, Department of Information \& Technology, SRM University, Chennai, India. \\ *Corresponding Author Email: ${ }^{1}$ maragathamhaarish@gmail.com, ${ }^{2}$ shobanak07@ gmail.com
}

\begin{abstract}
Sentiment analysis on Twitter data has paying more attention recently. The system's key feature, is the immediate communication with other users in an easy, fast way and user-friendly too. Sentiment analysis is the process of identifying and classifying opinions or sentiments expressed in source text. There is a huge volume of data present in the web for internet users and a lot of data is generated per second due to the growth and advancement of web technology. Nowadays, Internet has become best platform to share everyone's opinion, to exchange ideas and to learn online. People are using social network sites like facebook, twitter and it has gained more popularity among them to share their views and pass messages about some topics around the world. As tweets, notices and blog entries, the online networking is producing a tremendous measure of conclusion rich information. This client produced assumption examination information is extremely helpful in knowing the supposition of the general population swarm. At the point when contrasted with general supposition investigation the twitter assumption examination is much troublesome because of its slang words and incorrect spellings. Twitter permits 140 as the most extreme cutoff of characters per message. The two procedures that are mostly utilized for content examination is information base approach and machine learning approach. In this paper, we investigated the twitter created posts utilizing Machine Learning approach. Performing assumption examination in a particular area, is to distinguish the impact of space data in notion grouping. we ordered the tweets as constructive, pessimistic and separate diverse people groups' data about that specific space. In this paper, we developed a novel method for sentiment learning using the Spark coreNLP framework. Our method exploits the hashtags and emoticons inside a tweet, as sentiment labels, and proceeds to a classification procedure of diverse sentiment types in a parallel and distributed manner.
\end{abstract}

\section{Introduction}

Nowadays, people be likely to disseminate information, using short 140-character messages called "tweets", for various aspects on Twitter. Additionally, they follow other users in sequence to receive their status updates on tweets. In nature, Twitter has a wide distribution instant messaging platform and people are using that to get informed about world news, current scientific advancements, etc. Unavoidably, a variety of view clusters that includes a wealthy sentiment information is formed. Sentiment is termed as "A way of expressing one's thought, view, or attitude, particularly based mainly on emotion instead of reason" and it also describes someone's mood or critic towards a particular entity or domain $[3,5]$.

The information about overall sentiment tendency towards a specific topic may be used enormously in certain cases. For representation, assume a mechanical organization would be intrigued to think about their client's perspectives about the most recent item, with a specific end goal to get accommodating criticism that will use in the creation of the next device[6, 7]. In this way, clearly a comprehensive feeling examination for a day and age after the arrival of the new item is required. In addition [4, 6], client produced content that catches opinion data has turned out to be important among numerous web applications and data frameworks, for example, web crawlers or proposal systems. In the setting of this work, we use hash tags and emojis as supposition marks to perform characterization of different slant writes [23]. Hash tags are a tradition for including extra setting and metadata and are widely used in tweets. Their use is twofold: they give arrangement of a message as well as feature of a point and they enhance the seeking of tweets that allude to a typical subject. A hash tag is made by prefixing a word with a hash symbol (e.g. \#love). Emoji alludes to a computerized symbol or an arrangement of console images that serves to speak to an outward appearance, as :- ( for a pitiful face. Both, hashtags and emojis, give a fine-grained supposition learning at tweet level which makes them reasonable to be utilized for assessment mining.

The issue of opinion investigation has been contemplated widely amid late years. The greater part of existing arrangements is limited in brought together conditions and base on characteristic dialect handling strategies and machine learning approaches. Be that as it may, this sort of strategies are tedious and computationally serious $[16,22]$. Thus, it is restrictive to process in excess of a couple of thousand tweets without surpassing the abilities of a solitary server. Unexpectedly, a large number of tweets are distributed day by day on Twitter. Subsequently, underline arrangements are neither adequate nor appropriate for conclusion mining, since there is an enormous bungle between their preparing abilities and the exponential development of accessible information [16]. It is more than clear that there is a basic need to swing to high adaptable arrangements. Distributed computing innovations give instruments and foundation to make 
such arrangements and deal with the information distributed among various servers. The most conspicuous and strikingly effective instrument is the MapReduce programming model [7], created by Google, for handling extensive scale information. [13, 21]

\section{Preliminaries}

\subsection{Previous Work}

In spite of the fact that the idea of notion examination, or supposition mining, is generally new, the exploration around this area is very broad. Early examinations center around report level supposition investigation concerning motion picture or item audits $[11,30]$ and posts distributed on site pages or online journals [29]. Because of the multifaceted nature of record level supposition mining, numerous endeavors have been made towards the sentence level sentiment analysis. The arrangements exhibited in inspect expressions and dole out to every last one of them a conclusion extremity (positive, negative, nonpartisan) [25, 26, 28]. A less researched zone is the theme based assessment examination $[15,17]$ because of the trouble to give a satisfactory meaning of point and how to consolidate the slant factor into the conclusion mining undertaking. The most widely recognized ways to deal with go up against the issue of sentiment examination incorporate machine learning as well as characteristic dialect preparing procedures. In, the creators utilize [20] Naive Bayes, Maximum Entropy and Support Vector Machines to characterize film audits as positive or negative, and perform a correlation between the techniques as far as order execution. Then again, Nasukawa and Yi [18] endeavor to distinguish semantic connections between the estimation articulations and the subject. Together with a syntactic parser and an opinion vocabulary their approach figures out how to expand the precision of assumption investigation within web pages and online articles.

Also, Ding and Liu [8] describe a course of action of semantic standards together with another conclusion add up to ability to recognize incline presentations in online thing overviews. In the midst of the latest five years, Twitter has become much thought for suspicion examination. In [2], the makers proceed to a 2-step arrange process. In the underlying advance, they separate messages as subjective and objective and in the second step they perceive the subjective tweets as positive or negative. Davidov et al. [6] survey the dedication of different features (e.g. n-grams) together with a kNN classifier. They misuse the hashtags and smileys in tweets to portray suspicion classes and to avoid manual clarification. In this paper, we grasp this approach and staggeringly extend it to enable the examination of significant scale To twitter data. Agarwal et al. [1] examine the utilization of a tree piece model for distinguishing opinion introduction in tweets. A three-advance classifier is proposed in [12] that takes after an objective ward estimation order system. Besides, a diagram based model is proposed in [23] to perform assessment mining in Twitter information from a point based viewpoint. A later approach [27], fabricates a supposition and emoji vocabulary to help multi dimensional slant investigation of Twitter information. A vast scale arrangement is introduced in [14] where the creators assemble an opinion vocabulary and characterize tweets utilizing a MapReduce calculation and a disseminated database show. In spite of the fact that the precision of the technique is great, it experiences the tedious development of the supposition vocabulary. Our approach is substantially less complex and completely misuses the abilities of Spark system. To our best learning, we are the first to introduce a Spark-based extensive scale approach for conclusion mining on Twitter information without the need of building an assumption vocabulary or continuing to any manual information explanation.

\subsection{Spark Framework}

Apache Spark $[13,21]$ is a quick and general motor for extensive scale information handling. Basically, it is the advancement of Hadoop [10, 24] structure. Hadoop is the open source execution of the MapReduce demonstrate and is broadly utilized for conveyed preparing among various servers. It is perfect for cluster based procedures when we have to experience all information. Be that as it may, its execution drops quickly for certain issue writes (e.g. when we need to manage iterative or chart based calculations). Spark is a brought together pile of different firmly coordinated segments and conquers the issues of Hadoop. It has a Directed Acyclic Graph (DAG) execution motor that backings cyclic information stream and in-memory registering. Subsequently, it can run programs up to 100x quicker than Hadoop in memory, or 10x speedier on plate. Start incorporates a heap of libraries that consolidate SQL, gushing, machine learning and diagram handling in a solitary motor. Start offers some abnormal state systems, for example, reserving and makes simple to construct circulated applications in Java, Python, Scala and R. The applications are converted into MapReduce employments and keep running in parallel. Besides, Spark can get to various information sources, for example, HDFS or HBase [30].

\section{Sentiment Analysis Framework}

\subsection{Spark Core NLP}

Our pipeline framework was at first intended for inner utilize. Beforehand, when joining numerous characteristic dialect examination segments, each with their own specially appointed APIs, we had entwined them with custom paste code. The subsequent Annotation, containing all the examination data included by the Annotators, can be yield in XML or plain content forms. Annotation pipeline was produced in 2006 keeping in mind the end goal to supplant this scramble with something better. A uniform interface was accommodated an Annotator that includes some sort of examination data to some content. An Annotator does this by taking in an Annotation question which it can include additional data. An Annotation is put away as a sort safe heterogeneous guide, following the thoughts for this information write exhibited by Bloch (2008). This fundamental design has demonstrated very fruitful, is as yet the premise of the framework depicted here.

The inspirations were:

- To have the capacity to rapidly and effortlessly get semantic comments for a content.

- To shroud varieties crosswise over segments behind a typical API.

- To have a negligible theoretical impression, so the framework is anything but difficult to learn.

- To give a lightweight system, utilizing plain Java objects (as opposed to something of heavier weight, for example, XML or UIMA's Common Analysis System (CAS) objects).

In 2009, at first as a component of a multi-site give venture, the system was extended to be more easily usable by a more broad extent of customers. We gave a summon line interface and the ability to work out an Annotation in various courses of action, including XML. Moreover work provoked the structure being discharged as free open source programming in 2010. From one viewpoint, from a compositional perspective, Stanford Core NLP does not attempt to do everything. It is essentially a straight forward pipeline building. It gives only a Java API.1It does not attempt to give different machine scale-out (be that as it may it gives multi-strung preparing on a singular machine). It gives a straightforward solid API. Regardless, these essentials fulfill a generous level of potential customers, and the subsequent straightforwardness makes it less requesting for customers to begin with the framework. That is, the fundamental favored angle 
of Stanford CoreNLP over greater frameworks like UIMA (Ferrucci and Lally, 2004) or GATE (Cunningham et al., 2002) is that customers don't have to learn UIMA or GATE before they can get started; they simply need to know a little Java $[25,28]$.

Before long, this is a broad and basic differentiator. On the off chance that more personality boggling circumstances are required, for instance, different machine scale-out, they can frequently be refined by running the examination pipeline inside a system that spotlights on appropriated workflows(such as Hadoop or Spark). Diverse structures attempt to give all the more, for instance, the UIUC Curator(Clarke et al., 2012), which fuses cover machine client server correspondence for getting ready and the putting away of typical lingo examinations. However, this value incorporates some huge destructions.

The framework is unpredictable to introduce and complex to get it. In addition, by and by, an association may well be focused on a scale-out arrangement which is not the same as that gave by the characteristic dialect examination toolbox. For instance, they might utilize Kryo [30] or Google's proto yet for double serialization as opposed to Apache Thrift which underlies Curator. For this situation, the client is ideally serviced by a genuinely little and independent common dialect examination framework, instead of something which accompanies a great deal of things for a wide range of purposes, the greater part of which they are not utilizing. On the other hand, most customers advantage colossally from the game plan of a game plan of consistent, effective, high. Everything thought of it as, can call an examination fragment written in various vernaculars through a fitting wrapper Annotator, and hence, it has been wrapped by various people to give Stanford CoreNLP binds to various tongues. Quality semantic examination parts, which can be easily summoned for typical circumstances. While the designer of a greater structure may have settled on general arrangement choices, for instance, how to manage scale out, they are likely not going to be a NLP ace, and are consequently hunting down NLP sections that essentially work. This is a gigantic favored point of view that Stanford CoreNLP and GATE have over the empty instrument compartment of an Apache UIMA download, something kept an eye on some degree by the headway of all around consolidated part packages for UIMA, for instance, ClearTK (Bethard et al., 2014) [29], DKPro Core(Gurevych et al., 2007), and JCoRe (Hahn et al.,2008). Regardless, the plan gave by these groups remains harder to learn, more many-sided and heavier weight for customers than the pipeline depicted here.

Practically speaking, this is a huge and essential differentiator. In the event that more unpredictable situations are required, for example, different machine scale-out, they can ordinarily be accomplished by running the examination pipeline inside a framework that spotlights on dispersed workflows(such as Hadoop or Spark). Different frameworks endeavor to give all the more, for example, the UIUC Curator(Clarke et al., 2012), which incorporates bury machine customer server correspondence for preparing and the storing of characteristic dialect examinations. In any case, this usefulness includes some major disadvantages. The framework is perplexing to introduce and complex to get it. In addition, practically speaking, an association may well be focused on a scale-out arrangement which is unique in relation to that gave by the normal dialect investigation toolbox. For instance $[9,10]$, they might utilize Kryo or Google's proto yet for paired serialization as opposed to Apache Thrift which underlies Curator. For this situation, the client is ideally serviced by a genuinely little and independent regular dialect investigation framework, instead of something which accompanies a considerable measure of stuff for a wide range of purposes, the greater part of which they are not utilizing. Then again, most clients advantage extraordinarily from the arrangement of an arrangement of steady, powerful, high. In any case, it can call an examination part written in different dialects by means of a suitable wrapper Annotator, and thusly, it has been wrapped by numerous individuals to give Stanford CoreNLP ties to different dialects.
Quality semantic investigation segments, which can be effortlessly conjured for regular situations. While the manufacturer of a bigger framework may have settled on general outline decisions, for example, how to deal with scale out, they are probably not going to be a NLP master, and are consequently searching for NLP segments that simply work. This is an enormous favorable position that Stanford CoreNLP and GATE have over the void tool compartment of an Apache UIMA download, something tended to a limited extent by the advancement of all around incorporated part bundles for UIMA, for example, ClearTK (Bethard et al., 2014), DKPro Core(Gurevych et al., 2007) [21, 23], and JCoRe (Hahn et al.,2008). Be that as it may, the arrangement gave by these bundles stays harder to learn, more unpredictable and heavier weight for clients than the pipeline portrayed here.

The framework comes bundled with models for English. Isolate display bundles offer help for Chinese and for the case-obtuse handling of English. Support for different dialects is less total, yet a considerable lot of the Annotators likewise bolster models for French, German, and Arabic (see supplement B), and building models for facilitating dialects is conceivable utilizing the hidden instruments. In this area, we plot the gave annotators, concentrating on the English variants. It ought to be noticed that a portion of the model's basic annotators are prepared from explained corpora utilizing directed machine learning, while others are lead-based parts, which all things considered frequently require some dialect assets of their own. tokenize [2, 15] Tokenizes the content into an arrangement of tokens.

The English portion gives a PTB style tokenizer, extended to sensibly manage riotous and web content. The relating sections for Chinese and Arabic give word what's more, clitic division. The tokenizer saves the character adjusts of each token in the data content. Clean XML Removes most or all XML marks from the records split Splits a gathering of tokens into sentences certified case Determines the comprehensible authentic occurrence of tokens in content (that is, their possible case in particularly adjusted substance), where this information was lost, e.g., for all promoted content. This is completed with a discriminative model using a CRF course of action tagger (Finkel et al., 2005) [19, 22].

\subsection{Elasticsearch}

Elasticsearch is an Apache Lucene-based hunt server. It was created by Shay Banon and distributed in 2010. It is currently kept up by Elasticsearch BV. Its most recent variant is 2.1.0. Elasticsearch is a continuous conveyed and open source fullcontent hunt and examination motor. It is available from RESTful web benefit interface and utilizations pattern less JSON (JavaScript Object Notation) reports to store information. It is based on Java programming dialect, which empowers Elasticsearch to keep running on various stages. It empowers clients to investigate extensive measure of information at rapid.

The general highlights of Elasticsearch will be, Elasticsearch is versatile up to petabytes of organized and unstructured information. Elasticsearch can be utilized as a substitution of archive stores like MongoDB and RavenDB. Elasticsearch utilizes denormalization to enhance the hunt execution. Elasticsearch is one of the well known endeavor web crawlers, which is presently being utilized by numerous enormous associations like Wikipedia, The Guardian, Stack Overflow, GitHub and so on. Elasticsearch is open source and accessible under the Apache permit version 2.0.

\subsubsection{Elasticsearch - Key Concepts}

The key ideas of Elasticsearch are as per the following:

- Node: It alludes to a solitary running occurrence of Elasticsearch. Single physical and virtual server obliges various hubs relying on the abilities of their physical assets like RAM, Stack and processing power. 
- Cluster: It is an accumulation of at least one hubs. Cluster gives aggregate ordering what's more, look capacities over every one of the hubs for whole information.

- Index: It is an accumulation of various kind of reports and record properties. File additionally utilizes the idea of shards to enhance the execution. For instance, a set of report contains information of a person to person communication application.

- Type/Mapping: It is an accumulation of records sharing an arrangement of normal fields introduce in a similar record. For instance, an Index contains information of a social organizing application, and afterward there can be a particular kind for client profile information, another write for informing information and another for remarks information.

- Document: It is an accumulation of fields in a particular way characterized in JSON design. Each record has a place with a sort and lives inside a file. Each archive is related with a remarkable identifier, called the UID.

- Shard: Indexes are evenly subdivided into shards. This implies every shard contains every one of the properties of record, yet contains less number of JSON objects than list. The flat partition makes shard an autonomous hub, which can be store in any hub. Essential shard is the first even piece of a list and after that these essential shards are recreated into reproduction shards.

- Replicas: Elasticsearch enables a client to make copies of their lists and shards. Replication not just aides in expanding the accessibility of information if there should be an occurrence of disappointment, yet additionally enhances the execution of seeking via completing a parallel hunt activity in these reproductions.

\subsubsection{Elasticsearch - Advantages}

- Elasticsearch is created on Java, which makes it good on relatively every stage.
- Elasticsearch is ongoing, as it were following one moment the additional archive is accessible in this motor.

- Elasticsearch is appropriated, which makes it simple to scale and incorporate in any enormous

association.

- Creating full reinforcements are simple by utilizing the idea of the entryway, which is available in flexible hunt.

- Handling multi-tenure is simple in Elasticsearch when contrasted with Apache Solr.

- Elasticsearch utilizes JSON questions as reactions, which makes it conceivable to summon the versatile hunt server with countless programming dialects.

- Elasticsearch underpins relatively every report write with the exception of those that don't bolster content rendering.

\section{Proposed Framework}

In the first place, we got the labels from the tweets, check how often it (a tag) shows up and sort them by the tally. From that point forward, we hold on the outcome to point Splunk (or some other apparatus for this issue) to it. We could fabricate some intriguing dashboards utilizing this data so we can track the most slanting hashtags. In view of this data my associate could make battles and utilize these prominent labels to draw in a greater group of onlookers.

In the wake of trying different things with various applications to process spilling information like Spark streaming, flume, kafka, storm and so on gives now a chance to take a gander at how assumption scores can be created for tweets using Spark stanford CoreNLP and assemble representation dashboards on this information utilizing elasticsearch and kibana.

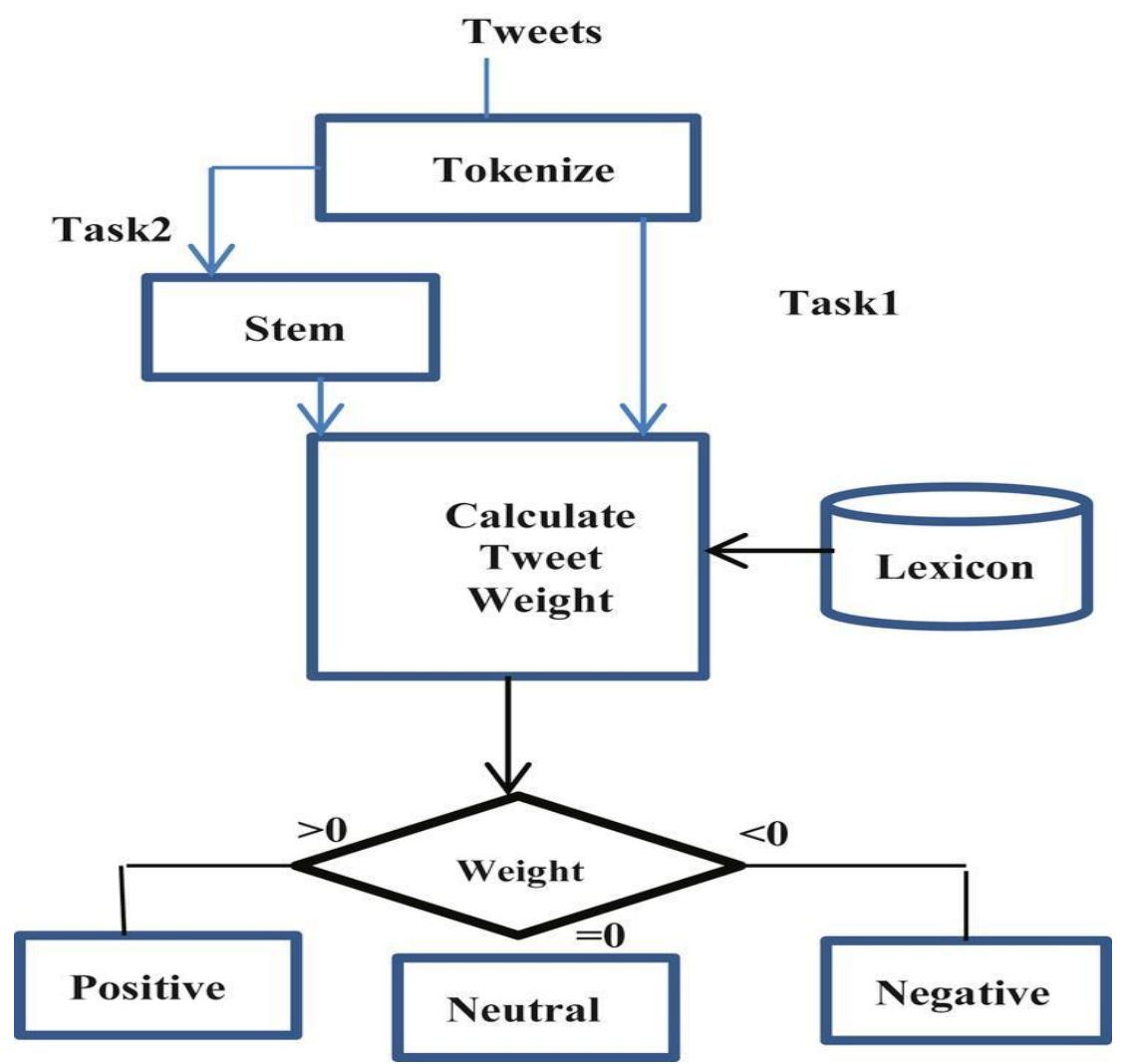

\section{Implementation}

This work is implemented using ApachSpark, First the tweets are collected from the twitter's official website. Once we register in twitter site, the latest tweets of about size $1 \mathrm{~GB}$ can be downloaded. The twitter site will generate security keys and oAuth tokens for every user, It is used mainly for coding in Spark. Next step is to create a scala maven project and the corresponding pom.xml file is updated with the dependencies that are required for this work. A scala object file was created, to receive the 
streaming data that are collected from the twitter. Using the twitter data, the sentiment scores are detected on each tweet by importing the package stanford coreNLP library. Once the analysis has been done, the output can be visualized by creating an index in elasticsearch and the ouput is written in that index. The index created in this paper is named as twitter_092517/tweet. Elasticsearch basically requires index content that can be translated into a document. Before storing the content in the created index (twitter 092517/tweet), each RDD in Spark is transformed to a Map object. The stanford university provided a useful natural language processing library coreNLP in Spark, to parse and detect the sentiments of each tweet data.

Stanford coreNLP gives a device pipeline as far as annotators utilizing which distinctive phonetic investigation instruments might be connected on content. Following annotators are incorporated into this case:

-tokenize - Divides content into an arrangement of words

-ssplit - Split the content into sentence. Distinguish fullstop. outcry and so forth and split sentences

-pos - Reads message and appoints parts of discourse to each word, for example, thing, verb, descriptive word, and so on. Ex. "This is a basic sentence" will be labeled as "This/DT is/VBZ a/DT test/NN sentence/NN"

-lemma - Group together types of a word so they can be examined as a solitary thing.

-parse - Provides syntactic investigation

-sentiment - Provides show for assumption investigation. Joins a binarized tree of the sentence. The hubs of the tree at that point contain the comments from RNNCoreAnnotations showing the anticipated class and scores for that subtree. The slant estimations of the individual words are accumulated at the base of the binarized tree.

Sentiment score is then found the middle value of in view of length of each sentence as longer sentence must convey more weight in the general opinion of the content.

\section{Results and Discussion}

Once the implementation is done, the results can be viewed visually by using Elastic search and Kibana. Using the index created twitter_092517/tweet before, the contents of output are transformed to ElasticSearch. From the total number of 4,315 tweets data, the Spark calculates sentiment score for each tweet data and based on the score it is classified as either positive, negative neutral, not understanding and very negative categories. In this given tweet data 4,315 tweets are classified into 3,249 of negative, 138 of positive, 847 of neutral, 75 of not understood and 8 of very negative categories.

The results are displayed below with total counts of tweet data for analysis and the list of classified tweets based one sentiments and the quarter hourly analysis of classified tweets and the pie chart view of classified tweets and the trending Hash tags list and the graphical view of text based sentiment classification. Thus this work of sentiment analysis using Spark coreNLP gives a clear visualization of classified tweets data and more accurate results with less time consuming.

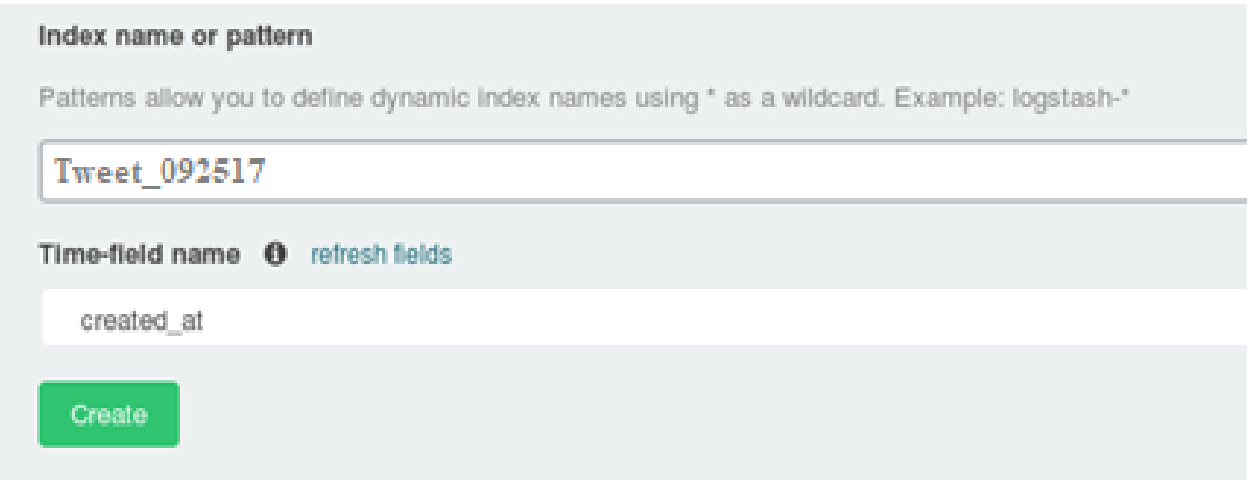

Fig. 2: Elastic search view of results based on the created index twitter_092517

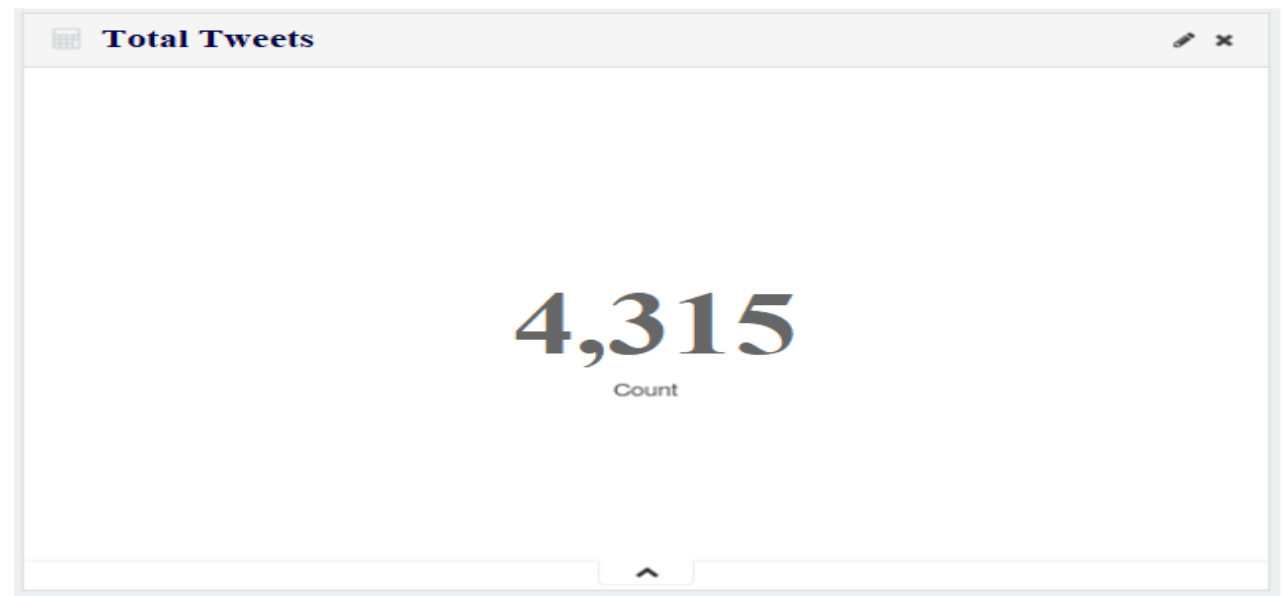

Fig. 3: The Total Tweets count for Analysis 


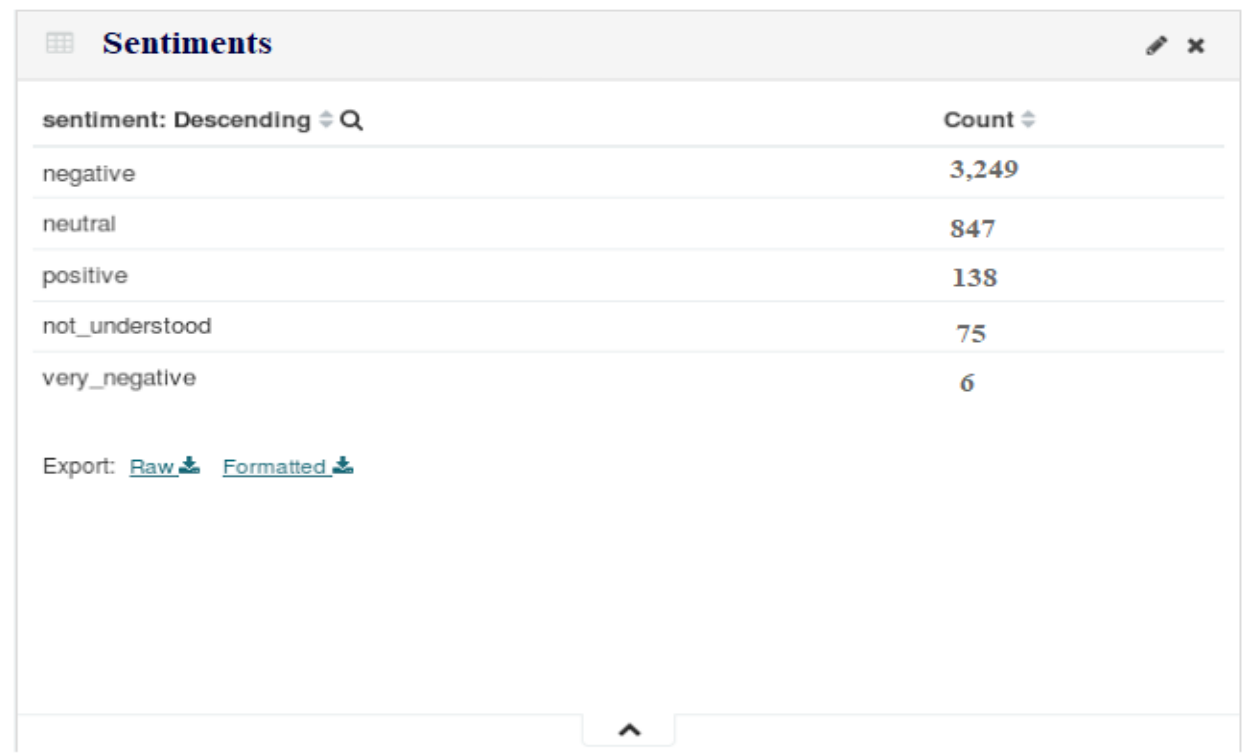

Fig. 4: List of classified Tweets based on Sentiments

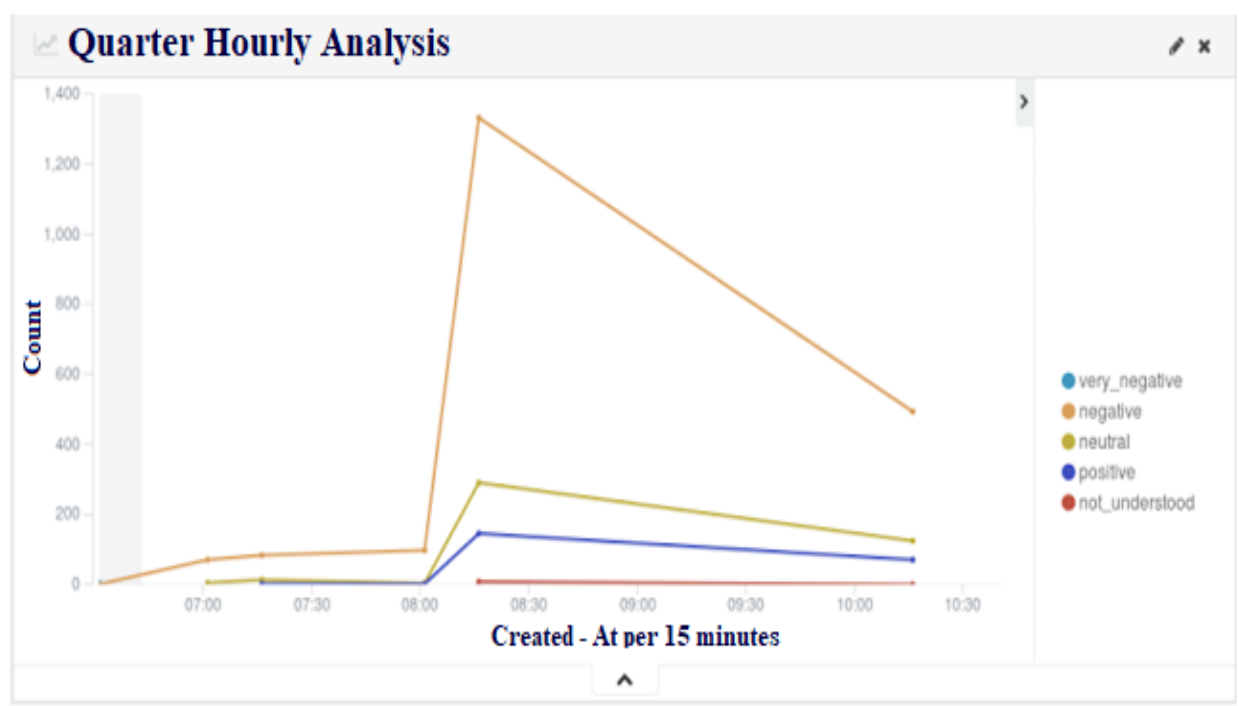

Fig. 5: Quarter Hourly Analysis of Classified Tweets

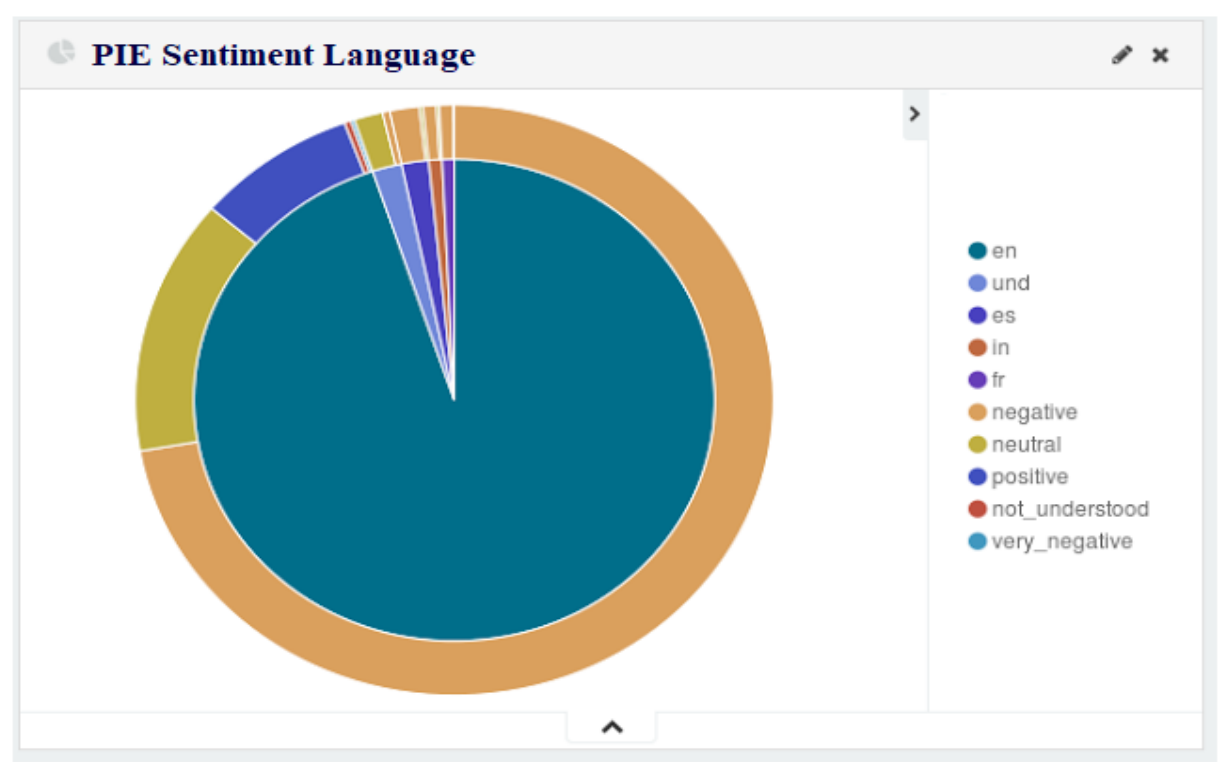

Fig. 6: The PIE Chart View of Classified Tweets 


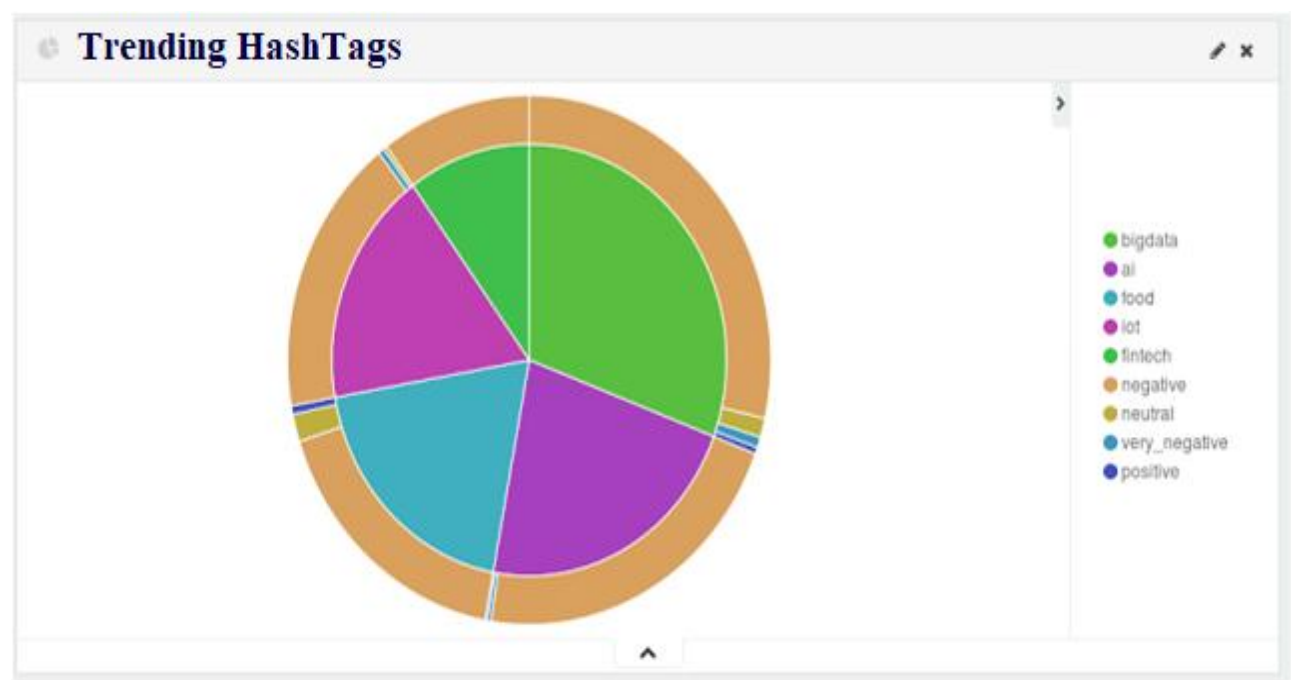

Fig. 7: Trending Hash Tags

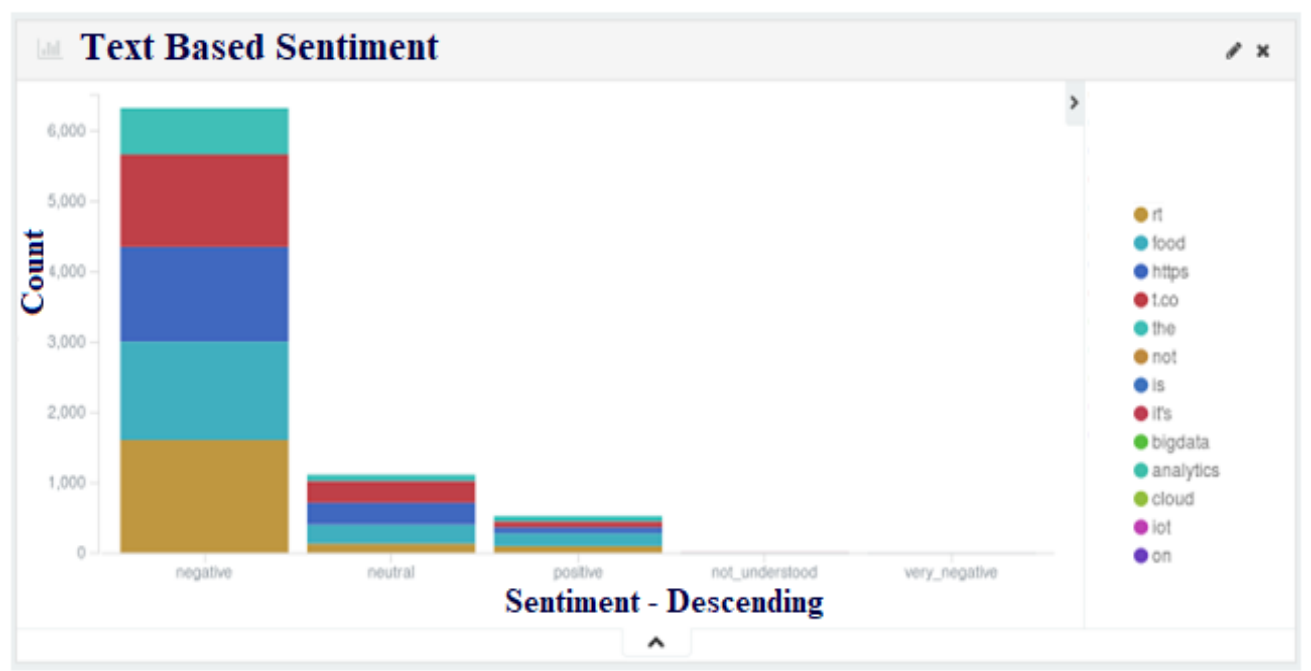

Fig. 8: The Graphical view of Text Based Sentiments

\section{Conclusion and Future work}

In the context of this work, we presented a novel technique for sentiment learning in the Spark framework and visualizing the results using Elasticsearch and Kibana. The proposed Stanford natural language processing library coreNLP is very efficient and most useful to process the text using the NLP functions and it helps us to classify the text based on sentiment scores. Since, this classification used a faster distributed and parallel computing engine framework Spark, the performance is much better compared to other works that are discussed before in this paper. The visualization frameworks Elasticsearch and Kibana are used to extract the output from Spark and it is visualized in different formats with the created index. So, the users can easily understood the results and can identify which sentiment (positive, negative and neutral) has received more tweets on that particular topic or domain. It also gives the most trending hashtags of that topic. In the near future, this work can be still extended for sentiment analysis with massive data in terms of Terabytes/Petabytes and can be distributed among multiple nodes of cluster to achieve less time consuming and more accurate results for large data sets. In addition to this, the tweets data can also be classified for multiple domains and the results can be visualized by comparing the different topics or domains. This would help in future to get more and efficient knowledge from the views that are posted by various kinds of people using this twitter application.

\section{References}

[1] Agarwal, B. Xie, I. Vovsha, O. Rambow, and R. Passonneau. Sentiment analysis of twitter data. In Proceedings of the Workshop on Languages in Social Media, pages 30\{38, 2011.

[2] L. Barbosa and J. Feng. Robust sentiment detection on twitter from biased and noisy data. In Proceedings of the 23rd International Conference on Computational Linguistics: Posters, pages 36\{44, 2010.

[3] H. Bloom. Space/time trade-o_s in hash coding with allowable errors. Commun. ACM, 13(7):422\{426, 1970.

[4] Z. Cheng, J. Caverlee, and K. Lee. You are where you tweet: A content-based approach to geo-locating twitter users. In Proceedings of the 19th ACM International Conference on Information and Knowledge Management, pages 759 \{768, 2010.

[5] Davidov and A. Rappoport. E_cient unsupervised discovery of word categories using symmetric patterns and high frequency words. In Proceedings of the 21st International Conference on Computational Linguistics and the 44th Annual Meeting of the Association for Computational Linguistics, pages 297\{304, 2006.

[6] D. Davidov, O. Tsur, and A. Rappoport. Enhanced sentiment learning using twitter hashtags and smileys. In Proceedings of the 23rd International Conference on Computational Linguistics: Posters, pages $241\{249,2010$.

[7] J. Dean and S. Ghemawat. Mapreduce: Simpli_ed data processing on large clusters. In Proceedings of the 6th Symposium on Operating Systems Design and Implementation, pages 137\{150, 2004.

[8] X. Ding and B. Liu. The utility of linguistic rules in opinion mining. In Proceedings of the 30th Annual International ACM 
SIGIR Conference on Research and Development in Information Retrieval, pages $811\{812,2007$.

[9] Hadoop. The apache software foundation: Hadoop homepage http://hadoop.apache.org/, 2015. [Online; accessed 20-September2015].

[10] M. Hu and B. Liu. Mining and summarizing customer reviews. In Proceedings of the Tenth ACM SIGKDD International Conference on Knowledge Discovery and Data Mining, pages 168\{177, 2004.

[11] L. Jiang, M. Yu, M. Zhou, X. Liu, and T. Zhao.Target-dependent twitter sentiment classi_cation. In Proceedings of the 49th Annual Meeting of the Association for Computational Linguistics: Human Language Technologies - Volume 1, pages 151\{160, 2011.

[12] H. Karau, A. Konwinski, P. Wendell, and M. Zaharia. Learning Spark: Lightning-Fast Big Data Analysis. O'Reilly Media, 2015.

[13] V. N. Khuc, C. Shivade, R. Ramnath, and J. Ramanathan. Towards building large-scale distributed systems for twitter sentiment analysis. In Proceedings of the 27th Annual ACM Symposium on Applied Computing, pages 59\{464, 2012.

[14] C. Lin and Y. He. Joint sentiment/topic model for sentiment analysis. In Proceedings of the 18th ACM Conference on Information and Knowledge Management, pages 375\{384, 2009.

[15] J. Lin and C. Dyer. Data-Intensive Text Processing with MapReduce. Morgan and Claypool Publishers, 2010.

[16] Q. Mei, X. Ling, M. Wondra, H. Su, and C. Zhai. Topic sentiment mixture: Modeling facets and opinions in weblogs. In Proceedings of the 16th International Conference on World Wide Web, pages $171\{180,2007$.

[17] T. Nasukawa and J. Yi. Sentiment analysis: Capturing favorability using natural language processing. In Proceedings of the $2 \mathrm{Nd}$ International Conference on Knowledge Capture, pages $70\{77$, 2003.

[18] N. Nodarakis, E. Pitoura, S. Sioutas, A. K. Tsakaidis, D. Tsoumakos, and G. Tzimas. kdann+: A rapid aknn

[19] classi_er for big data. T. Large-Scale Data- and KnowledgeCentered Systems, 23:139\{168, 2016.

[20] B. Pang, L. Lee, and S. Vaithyanathan. Thumbs up?: Sentiment classi_cation using machine learning

[21] techniques. In Proceedings of the ACL-02 Conference on Empirical Methods in Natural Language Processing - Volume 10, pages $79\{86,2002$.

[22] Spark. The apache software foundation: Spark homepage http://spark.apache.org/, 2015. [Online; accessed 27-December2015].

[23] M. van Banerveld, N. Le-Khac, and M. T. Kechadi. Performance evaluation of a natural language processing approach applied in white collar crime investigation. In Future Data and Security Engineering - First International Conference, FDSE 2014, Ho Chi Minh City, Vietnam, November 19-21, 2014, Proceedings, pages $29\{43,2014$

[24] X. Wang, F. Wei, X. Liu, M. Zhou, and M. Zhang. Topic sentiment analysis in twitter: A graph-based hashtag sentiment classi_cation approach. In Proceedings of the 20th ACM International Conference on Information and Knowledge Management, pages $1031\{1040,2011$

[25] T. White. Hadoop: The De nitive Guide, 3rd Edition. O'Reilly Media / Yahoo Press, 2012.

[26] T. Wilson, J. Wiebe, and P. Ho_mann. Recognizing contextua polarity in phrase-level sentiment analysis. In Proceedings of the Conference on Human Language Technology and Empirical Methods in Natural Language Processing, pages 347 \{354, 2005

[27] T. Wilson, J. Wiebe, and P. Ho_mann. Recognizing contextual polarity: An exploration of features for phrase-level sentiment analysis. Comput. Linguist., 35(3):399 \{433, Sept. 2009.

[28] Y. Yamamoto, T. Kumamoto, and A. Nadamoto. Role of emoticons for multidimensional sentiment analysis of twitter. In Proceedings of the 16th International Conference on Information Integration and Web-based Applications \&\#38; Services, pages 107\{115, 2014.

[29] H. Yu and V. Hatzivassiloglou. Towards answering opinion questions: Separating facts from opinions and identifying the polarity of opinion sentences. In Proceedings of the 2003 Conference on Empirical Methods in Natural Language Processing, pages $129\{136,2003$.

[30] W. Zhang, C. Yu, and W. Meng. Opinion retrieval from blogs. In Proceedings of the Sixteenth ACM Conference on Conference on Information and Knowledge Management, pages 831 \{840, 2007.

[31] L. Zhuang, F. Jing, and X.-Y. Zhu. Movie review mining an summarization. In Proceedings of the 15th ACM International
Conference on Information and Knowledge Management, pages $43\{50,2006$ 